Die Mitfinanzierung der deutschen Übersetzung der überarbeiteten Version des «Appraisal of Guidelines for Research and Evaluation» (AGREE II) war Anfang 2013 einer der ersten offiziellen Unterstützungsanträge an die Schweizerische Akademie für Qualität in der Medizin SAQM. Unterdessen liegt die übersetzte Neuauflage dieses international anerkannten Instruments zur Beurteilung der methodologischen Qualität im Rahmen der Entwicklung und Anwendung medizinischer Leitlinien (Guidelines) vor. Die Unterstützung ist eine konsequente Fortsetzung des bereits 2001 getätigten Engagements seitens der FMH, als es um die Erstellung und Publikation der ersten deutschsprachigen Version (AGREE I) ging. Mit dem erneuten Engagement leistet die FMH/SAQM einen Beitrag dazu, dass ein niederschwelliger Zugang in deutscher Sprache auch für die vorliegende Weiterentwicklung des Instruments sichergestellt ist.

Dr. med. Christoph Bosshard, Mitglied des Zentralvorstandes der FMH, Departementsverantwortlicher Daten, Demographie und Qualität, Schweizerische Akademie für Qualität in der Medizin SAQM

\title{
Deutsche Version des AGREE II
}

\author{
AGREE (Appraisal of Guidelines for REsearch and Evaluation) dient einerseits zur \\ Qualitätsbeurteilung von klinischen Leitlinien und andererseits als Leitfaden bei der \\ Entwicklung von Leitlinien. Die 2. Version wurde gemäss international anerkannten \\ Richtlinien übersetzt und steht auf der Homepage des AGREE-Trusts zur Verfügung.
}

\author{
Anne-Kathrin Rausch Osthoffa, \\ Bernhard Aebischer ${ }^{b}$, \\ Ulrich Siering $c$ \\ a Forschungsstelle Physiothera- \\ pie, Zürcher Hochschule für \\ Angewandte Wissenschaften \\ b Berner Fachhochschule \\ c Institut für Qualität und \\ Wirtschaftlichkeit im \\ Gesundheitswesen Köln
}

Das Appraisal of Guidelines for REsearch and Evaluation (AGREE) ist der internationale Goldstandard bei der Beurteilung der methodologischen Qualität im Rahmen der Entwicklung und Anwendung medizinischer Leitlinien [1]. Medizinische Leitlinien sind «systematisch entwickelte Aussagen, die den Behandelnden und den Patienten helfen sollen, Entscheidungen über eine angemessene Gesundheitsversorgung bei bestimmten klinischen Zuständen zu treffen» [2]. Auch in der Gesundheitspolitik [3] und Gesundheitsversorgung (Gesundheitsförderung, Screening, Diagnostik) spielen Leitlinien eine wichtige Rolle. Quellen für Leitlinien sind u.a. www.ahrq.gov, www.clinicalguidelines.gov.au, www.aezq.de, www.g-i-n.net.

Evidenzbasierte Leitlinien sind essentiell, um sowohl Klinikern als auch Patienten die bestmögliche medizinische Versorgung aufzuzeigen [4]. Die Qualität der Leitlinie bedingt die Zuverlässigkeit der Empfehlungen [3]. Angemessene Methoden und deren konsequente Handhabung während der Leitlinienentwicklung sind wichtig für eine erfolgreiche Implementierung der resultierenden Empfehlungen [5]. Zur Implementierung und niedrigschwelligen Anwendung des AGREE II im deutschsprachigen Raum ist die Übersetzung des englischen Originals notwendig.

Die Forschungsstelle Physiotherapiewissenschaften der Zürcher Hochschule für Angewandte Wissenschaften (ZHAW) hat in Zusammenarbeit mit dem Institut für Qualität und Wirtschaftlichkeit im Gesundheitswesen Köln (IQWiG) und der Berner Fachhochschule (BFH) diese deutsche Übersetzung des AGREE II, nach den Vorgaben des AGREE Trust, durchgeführt. Basierend auf den Vorgaben durch Beaton [6] gliederte sich das methodische Vorgehen in fünf Schritte: 1) Übersetzung des englischen Originals ins Deutsche durch zwei unabhängige Muttersprachler. 2) Konsentierung der beiden deutschen Versionen durch eine Arbeitsgruppe. 3) Rückübersetzung der ersten deutschen Version zurück ins Englische. Dieser Schritt wurde durch zwei unabhängige Muttersprachler vollzogen, die in dem bisherigen Arbeitsprozess nicht involviert waren. 4) Diskrepanzen zwischen dem englischen Original und der Rückübersetzung basierend auf der ersten deutschen Version wurden analysiert und die entsprechenden Textstellen überarbeitet. 5) Die daraus resultierende Version der deutschen Übersetzung wurde verschiedenen Experten für ein kritisches Feedback vorgelegt. Basierend auf dieser Rückmeldung wurde das Manuskript erneut überarbeitet. Abschliessend wurde eine Rechtschreibprüfung und Anpassung des Formats vorgenommen.

Das Projekt wurde durch den Verband der Schweizer ErgotherapeutInnen und die FMH/Schweizer Akademie für Qualität in der Medizin SAQM finanziell unterstützt. Die deutsche Übersetzung des AGREE II steht unter folgendem Link kostenlos zur Verfügung: www. agreetrust.org/resource-centre/agree-ii-translations/.

1 AGREE Collaboration. Development and validation of an international appraisal instrument for assessing the quality of clinical practice guidelines: the AGREE project. Qual Saf Health Care. 2003;12(1):18-23.

2 Woolf SH, Grol R, et.al. Clinical guidelines: potential benefits, limitations, and harm of clinical guidelines. BMJ. 1999;318(7182):527-30.

3 Committee to Advice the Public Health Service on Clinical Practice Guidelines, Institute of Medicine. In: Field MJ, Lohr KN, editors. Clinical practice guidelines: directions for a new program. Washington (DC): National Academy Press; 1990.

4 Brouwers C, Kho MW, et. al. Development of the AGREE II, part 1: performance, usefulness and areas for improvement. CMAJ. 2010;182(10):1045-52.

5 Shaneyfelt TM, Mayo-Smith MF, et al. Are guidelines following guidelines? The methodological quality practice guidelines in the peer- reviewed medical literature. JAMA. 1999;281(20):1900-5.

6 Beaton D, Bombardier C, et al. Recommendations for the cross-cultural adaptation of health status measures. Rosemont, IL: American Academy of Orthopedic Surgeons; 2002. 